\title{
ACYCLIC MODELS AND EXCISION
}

\author{
ROLF SCHÖN
}

\begin{abstract}
This note gives a proof of the excision theorem in singular homology using the method of acyclic models and avoiding the commonly used barycentric subdivision operators.
\end{abstract}

1. Notations, definitions. TopCov is the category of pairs $(X, \mathfrak{u})$, where $X$ is a topological space and $\mathfrak{U}$ a covering of $X$ having an open refinement. A morphism $f$ from $(X, \mathfrak{U})$ to $(Y, \mathfrak{B})$ is a map $f: X \rightarrow Y$ such that for every $U \in \mathfrak{U}$ we have $f(U) \subset V$ for some $V \in \mathfrak{B}$. Two maps $f, g:(X, \mathfrak{u}) \rightarrow(Y, \mathfrak{B})$ are called homotopic, $f \simeq g$, if there exists a homotopy $H: X \times[0,1] \rightarrow Y$ from $f$ to $g$ such that for every $U \in \mathfrak{U}$, we have $H(U \times[0,1]) \subset V$ for some $V \in \mathfrak{B}$. The definitions of a homotopy equivalence and of a strong deformation retract $(X, \mathfrak{U}) \simeq(Y, \mathfrak{B})$ are the obvious ones. $S(X, \mathfrak{U})$ is the subcomplex of the singular chain complex $S(X)=S(X, \mathfrak{I})$ generated by the singular simplexes $\sigma: \Delta \rightarrow X$ such that $\sigma(\Delta) \subset U$ for some $U \in \mathfrak{U}$. $\mathfrak{I}=\{X\}$ is the trivial covering. Using the naturality of the chain homotopy $D: S\left(j_{0}\right)$ $\simeq S\left(j_{1}\right): S(X) \rightarrow S(X \times[0,1])$ with $j_{i}=(x \mapsto(x, i))$ for $i=0$, 1, we easily get the following lemma and corollary.

2. Lemma. If $f \simeq g:(X, \mathfrak{U}) \rightarrow(Y, \mathfrak{B})$, then

$$
S(f) \simeq S(G): S(X, \mathfrak{u}) \rightarrow S(Y, \mathfrak{B}) .
$$

3. Corollary. A homotopy equivalence $f:(X, \mathfrak{U}) \rightarrow(Y, \mathfrak{B})$ induces a chain equivalence $S(f): S(X, \mathfrak{U}) \rightarrow S(Y, \mathfrak{B})$.

4. Lemma. For every object $(\square, \mathfrak{u})$ in TopCov with $\square$ an unit cube of any finite dimension, we have $H_{n}(\square, \mathfrak{u}) \cong H_{n}(*, \mathfrak{I})$ for $n \geqslant 0$.

Proof. We construct a finite sequence $\left(X_{i}, \mathfrak{U}_{i}\right), i=0, \ldots, k$, of objects in TopCov, such that $\left(X_{0}, \mathfrak{U}_{0}\right)=(\square, \mathfrak{u}),\left(X_{k}, \mathfrak{U}_{k}\right)=(*, \mathfrak{I})$, with $*$ one point and $\left(X_{i}, \mathfrak{U}_{i}\right)$ a strong deformation retract of $\left(X_{i-1}, \mathfrak{U}_{i-1}\right)$. By the Lebesgue covering lemma the cube $\square$ may be subdivided by sections parallel to its faces into a finite number, say $k$, of subcubes, each of which is contained in some $U \in \mathfrak{U}$. Now, beginning in one corner, we retract off one subcube after the other onto that part of its boundary which is in common with the remaining ones. The last cube is retracted onto a corner. For illustration we give the following picture of a subdivided two dimensional cube.

Received by the editors July 15, 1975.

AMS (MOS) subject classifications (1970). Primary 55B10; Secondary 55B40, 18 H05.

Key words and phrases. Excision axiom, singular homology, acyclic models. 


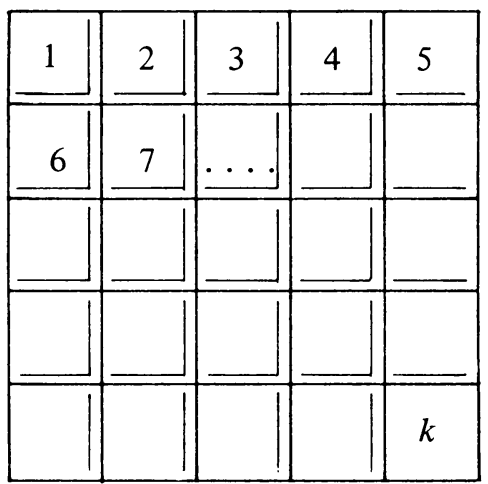

The subcubes $1,2,3, \ldots$ are successively retracted onto that part of boundary which is marked by a double line. That is, we define $X_{i}$ as $X_{i-1}$ with the $i$ th subcube retracted off and $\mathfrak{U}_{i}$ as $\mathfrak{U}_{i-1} \cap X_{i}$. Obviously $\left(X_{i}, \mathfrak{U}_{i}\right)$ is a strong deformation retract of $\left(X_{i-1}, \mathfrak{u}_{i-1}\right)$ in the sense of the homotopy notion in TopCov defined above. Corollary 3 gives the rest.

5. THEOREM. The inclusion

$$
i: S(X, \mathfrak{u}) \rightarrow S(X)
$$

is a chain equivalence.

Proof. We consider both sides of $(\S)$ as functors from TopCov to the category of the augmented chain complexes of abelian groups: $S_{0}(X, \mathfrak{u})$ $=S(X, \mathfrak{U}), S_{1}(X, \mathfrak{u})=S(X)$. As models in TopCov we choose all pairs $(\Delta, \mathfrak{B})$ with $\Delta$ a standard simplex of any dimension and $\mathfrak{B}$ a covering of $\Delta$ having an open refinement. Because of 4 and the homeomorphism $\Delta \simeq \square$, both functors are acyclic on models. The forgetful functor TopCov $\rightarrow$ Top induces a retraction $r: F \rightarrow S_{1}$, where $F(X, \mathfrak{U})$ is the free functor defined as the free abelian group generated by the set $\{\sigma:(\Delta, \mathfrak{B}) \rightarrow(X, \mathfrak{H}) /(\Delta, \mathfrak{B})$ model $\}$ with base the identities $\mathrm{id}_{\mathfrak{B}}:(\Delta, \mathfrak{B}) \rightarrow(\Delta, \mathfrak{B})$. The inclusion $i: S_{1}(X, \mathfrak{U})$ $\rightarrow F(X, \mathfrak{U})$ is given by considering $\sigma: \Delta \rightarrow X$ as $\sigma:\left(\Delta, \sigma^{-1} \mathfrak{U}\right) \rightarrow(X, \mathfrak{U})$, and we have clearly $r i=\mathrm{id}$. The functor $S_{0}$ is free by itself: in dimension $n$ we take the single element id: $(\Delta, \mathfrak{I}) \rightarrow(\Delta, \mathfrak{I}), \Delta$ the standard $n$-simplex, as base. Now the theorem on acyclic models applies; see 4.3.4 of [2, p. 169] and 11.11.1 of $[1$, p. 177].

6. REMARK. As in [2, p. 189] we get the excision isomorphism

$$
H_{*}(X-U, A-U) \cong H_{*}(X, A) \text { if } \bar{U} \subset \text { int } A,
$$

because $\{$ int $A, X-\bar{U}\}$ is an open refinement of $\{A, X-U\}$.

\section{REFERENCES}

1. A. Dold, Lectures on algebraic topology, Springer-Verlag, Berlin and New York, 1972.

2. E. H. Spanier, Algebraic topology, McGraw-Hill, New York, 1966. MR 35 \# 1007.

Mathematisches Institut der Universität, 69 Heidelberg 1, Im Neuenheimer Feld 288, West Germany 